\title{
$J$-HOLOMORPHIC DISCS AND REAL ANALYTIC HYPERSURFACES
}

\author{
WILLIAM ALEXANDRE AND EMMANUEL MAZZILLI
}

\begin{abstract}
We give in $\mathbb{R}^{6}$ a real analytic almost complex structure $J$, a real analytic hypersurface $M$ and a vector $v$ in the Levi null set at 0 of $M$, such that there is no germ of $J$-holomorphic disc $\gamma$ included in $M$ with $\gamma(0)=0$ and $\frac{\partial \gamma}{\partial x}(0)=v$, although the Levi form of $M$ has constant rank. Then for any hypersurface $M$ and any complex structure $J$, we give necessary conditions under which there exists such a germ of disc.
\end{abstract}

\section{INTRODUCTION}

Throughout all this paper, we denote by $M$ be a real analytic hypersurface in $\mathbb{R}^{2 n}$ such that 0 belongs to $M$. We denote by $\varphi$ a real analytic function such that $M=\{z \in$ $\left.\mathbb{R}^{2 n}, \varphi(z)=0\right\}$ and such that $d \varphi$ does not vanish on $M$. We also denote by $T M$ the tangent vector bundle to $M$.

We equip $\mathbb{R}^{2 n}$ with a real analytic almost complex structure $J$, i.e. a linear map from $T \mathbb{R}^{2 n}$ into itself such that $J^{2}=-I d$. Without restriction, we can always assume that $J(0)=J_{0}$, the standard complex structure in $\mathbb{R}^{2 n}=\mathbb{C}^{n}$, and that $J$ is as close as we need to $J_{0}$. We denote by $T^{J} M$ the $J$ invariant part of $T M$, that is $T^{J} M=T M \cap J T M$.

The Levi form $\mathcal{L}_{\varphi}$ of $M$ and its kernel are two objects linked to the geometry of $M$. The Levi form is defined for every vector field $X \in T^{J} M$ by

$$
\mathcal{L}_{\varphi}(X)=d \varphi(J[X, J X]) .
$$

We will also consider $\hat{\mathcal{L}}_{\varphi}$, the polar form of $\mathcal{L}_{\varphi}$ :

$$
\hat{\mathcal{L}}_{\varphi}(X, Y)=d \varphi(J[X, J Y]+J[Y, J X])+i d \varphi(J[X, Y]+J[J X, J Y]) .
$$

Both $\mathcal{L}_{\varphi}$ and $\hat{\mathcal{L}}_{\varphi}$ do not depend on the derivatives of $X$ and $Y$. Moreover, they depend on the defining function $\varphi$ up to multiplication by a nonnegative function. Therefore $\operatorname{ker} \mathcal{L}_{\varphi}$ does no depend on $\varphi$ and we simply denote it by ker $\mathcal{L}$.

When $J$ is integrable, or more simply when $J$ is the standard structure, and when ker $\mathcal{L}$ is a subbundle of $T^{J} M$, Freeman proved in 2] that there exists a complex foliation of $M$. However, when $J$ is a generic almost complex structure, there is no foliation by $J$ holomorphic manifolds of dimension greater than or equal to 2 because such objects do not exist in general. Hence Freeman's theorem does not hold anymore in the non integrable case; we can only hope to find a $J$-holomorphic disc with prescribed derivative of order 1 included in $M$. A $J$-holomorphic disc is a $C^{1}$ map $\gamma: \mathbb{D} \rightarrow \mathbb{R}^{2 n}$, where $\mathbb{D}$ is the unit disc

2000 Mathematics Subject Classification. 32Q60, 32Q65.

The first author is partially supported by A.N.R. BL-INTER09-CRARTIN. 
of $\mathbb{C}$ equipped with the standard structure $J_{0}$, such that for all $\zeta \in \mathbb{D}$, we have as linear map:

$$
d \gamma(\zeta) \circ J_{0}=J(\gamma(\zeta)) \circ d \gamma(\zeta)
$$

When $\gamma$ is a $J$-holomorphic disc included in $M$, i.e. $\gamma(\mathbb{D}) \subset M$, the vector $\frac{\partial \gamma}{\partial x}(0)$ belongs to $(\operatorname{ker} \mathcal{L})_{\gamma(0)}$. When $J$ is integrable and $\operatorname{ker} \mathcal{L}$ is a subbundle of $T^{J} M$, Freeman's result implies that the converse is true (even in the non real analytic case); that is, for any point $p \in M$ and any $v \in \operatorname{ker} \mathcal{L}$, there exists a $J$-holomorphic disc $\gamma$ included in $M$ such that $\gamma(0)=p$ and $\frac{\partial \gamma}{\partial x}(0)=v$. This result was generalized by Kruzhilin and Sukhov in [5] when $J$ is not integrable and $M$ is Levi flat, that is when $\operatorname{ker} \mathcal{L}=T^{J} M$. It was then natural to hope that this result was true in the real analytic case when $\operatorname{ker} \mathcal{L}$ is only a subbundle of $T^{J} M$. However, we will show that this is not the case, even when $M$ is pseudoconvex. We will prove in Section 2 the following theorem:

Theorem 1.1. There exist a real analytic almost complex structure $J$, a real analytic hypersurface $M$ in $\mathbb{R}^{6}$ such that $\operatorname{ker} \mathcal{L}$ is a subbundle of dimension 1 of $T^{J} M$ and a vector $v \in \operatorname{ker} \mathcal{L}_{0}$ such that no J-holomorphic disc $\gamma$, satisfying $\frac{\partial \gamma}{\partial x}(0)=v$, is tangent to $M$ at 0 at order greater than 5 .

The following natural question then arises: under which condition on $v \in T_{0}^{J} M$ does there exist a $J$-holomorphic disc $\gamma$ included in $M$ such that $\gamma(0)=0$ and $\frac{\partial \gamma}{\partial x}(0)=v$ ?

In order to answer this question, we fix a symmetric connexion $\nabla$ and for simplicity's sake, we use a multiplicative notation: when $X$ and $Y$ are two vector fields, we denote by $X . Y$ the vector field $\nabla_{X} Y$. We also introduce the following notations:

Notation 1.2. If $X$ is a vector field and $p$ and $q$ are two integers, we define the vector field $D_{X}^{p, q}$ by the formula

$$
D_{X}^{k, l} \phi=\underbrace{J X \cdot(J X \cdots(J X}_{l} \cdot \underbrace{(X \cdot(X \ldots(X}_{k} \cdot \phi) \ldots) .
$$

Notation 1.3. Let $X$ be a vector field. We denote by $\mathbb{L}(X)$ the complex Lie algebra generated by $X$, i.e. the smallest complex Lie algebra which contains $X$ and $J X$ and is such that for $Y$ and $Z$ in $\mathbb{L}(X),[Y, Z]$ belongs to $\mathbb{L}(X)$.

We will establish two sufficient conditions on a vector $X$ under which there exists a $J$-holomorphic disc $\gamma$ included in $M$ such that for all $k \in \mathbb{N}^{*}, \frac{\partial^{k} \gamma}{\partial x^{k}}(0)=D_{X}^{k-1,0} X(0)$. The first one is given by the following theorem:

Theorem 1.4. Let $X \in T^{J} M$ be a real analytic vector field in a neighborhood of $0 \in M$ such that $\mathbb{L}(X)$ is included in $T^{J} M$.

Then, for all $p \in M$ in a neighborhood of 0 , there exists a germ of J-holomorphic disc $\gamma$ such that

(1) $\gamma(0)=p$,

(2) for all $k \in \mathbb{N}^{*}, \frac{\partial^{k} \gamma}{\partial x^{k}}(0)=D_{X}^{k-1,0} X(p)$.

Unlike Freeman's Theorem [2], Theorem 1.4 makes no assumption on the rank of the Levi form $\mathcal{L}$. In particular, Theorem 1.4 can be applied to an integrable complex structure when $\operatorname{ker} \mathcal{L}$ is not a subbundle. In this case, we will show that for any $X \in \operatorname{ker} \mathcal{L}$, there 
exists a $J$-holomorphic disc $\gamma$ included in $M$ such that $\frac{\partial \gamma}{\partial x}(0)=X(0)$ (see Theorem 4.4). We will also give an example of application of our result in a non-integrable case and where the Levi form has constant rank (see Example 4.5).

In order to state our next result which requires a condition in the spirit of Freeman's theorem, we recall the following definition introduced in [1]:

Definition 1.5. We say that a vector field $X$ commutes at order $k$ at a point $p$ if for all $l \leq k$, all $X_{1}, \ldots, X_{l} \in\{X, J X\}$,

$$
\left[X_{1},\left[\ldots,\left[X_{l-1}, X_{l}\right] \ldots\right](p)=0 .\right.
$$

We will prove the following result:

Theorem 1.6. Let $\mathbb{L}$ be a subbundle of $T^{J} M$ such that the following property is true: if $X \in \mathbb{L}$ commutes at order $k$ at the point 0 , then for all $X_{1}, \ldots, X_{k+1} \in\{X, J X\}$, $\left[X_{1},\left[\ldots,\left[X_{k}, X_{k+1}\right] \ldots\right]\right](0)$ belongs to $\mathbb{L}_{0}$.

Then for all $X \in \mathbb{L}$, there exists a germ of J-holomorphic disc $\gamma$ such that $\gamma(0)=0$, $\frac{\partial^{k} \gamma}{\partial x^{k}}(0)=D_{X}^{k-1,0} X(0)$ for all $k \in \mathbb{N}^{*}$ and $\gamma(\mathbb{D}) \subset M$.

This Theorem 1.6 enables us to prove, in the real analytic case and in a constructive way, Kruzhilin and Suhkov's theorem [5] (See Theorem 4.7). We will also give examples of applications of Theorem 1.6 (see Example 4.8).

In order to prove Theorem 1.4 and 1.6. we prove a generalization of the existence results of $J$-holomorphic discs with prescribed jets of finite order, due to Sikorav in the case of 1jets (see [7]) and to Ivashkovich and Rosay [3] and independently to Barraud and Mazzilli [1] in the case of $k$-jets, for $k$ finite. For this, a key point is to show that if $\left(x_{k}\right)_{k \in \mathbb{N}}$ is an infinite sequence of vectors in $\mathbb{R}^{2 n}$ such that the series $\sum_{k} \frac{k+1}{k !}\left|x_{k}\right|$ converges, then there is a germ of $J$-holomorphic disc $\gamma$ included in $M$ such that $\frac{\partial^{k} \gamma}{\partial x^{k}}(0)=x_{k}$ for all $k \in \mathbb{N}$ (see Theorem 3.1 and Corollary 3.31). The general procedure to prove this is in some sense analogue to the case of finite jets but the proof is more intricate: we have to prove that some maps between two Banach spaces $\tilde{\Omega}$ and $\Omega$ are invertible in a neighborhood of the origin. In the case of $k$-jets, the Banach spaces considered were simply $\tilde{\Omega}=\left(\mathbb{R}^{2 n}\right)^{k}$ and $\Omega=C^{k+\alpha}(\overline{\mathbb{D}})$ for $\alpha>0$, with the $k$-jet belonging to $\left(\mathbb{R}^{2 n}\right)^{k}$ and the disc to $C^{k+\alpha}(\overline{\mathbb{D}})$. The first difficulty we have to overcome is to find the appropriate Banach spaces. Here, the $k$-jet becomes an infinite sequence of vectors of $\mathbb{R}^{2 n}$, so we have to consider a space of sequences with an appropriate norm. This role will be played by the space $\tilde{\Omega}$ of all sequences $\left(x_{k}\right)_{k}$ of vectors in $\mathbb{R}^{2 n}$ such that the norm $\left\|\left(x_{k}\right)_{k}\right\|_{\sim}:=\sum_{k} \frac{k+1}{k !}\left|x_{k}\right|$ is finite. The space of functions will be the space $\Omega$ of real analytic functions $f=\sum_{k, l} f_{k, l} z^{k} \bar{z}^{l}$ on the disc $\mathbb{D}$ such that $\|f\|:=\sum_{k, l}(1+k+l+k l)\left|f_{k l}\right|$ is finite. Then, as in the case of $k$-jets, we associate to any jet a function and to any function a jet (See Theorem 3.1 for details). Showing that these maps are firstly well defined and secondly invertible, we find a $J$-holomorphic disc with prescribed derivatives of any order.

In Proposition 3.4 we will prove that if $X$ is a real analytic vector field in a neighborhood of 0 , then the sequence $\left(D_{X}^{k-1,0} X(0)\right)_{k \in \mathbb{N}^{*}}$ belongs to $\tilde{\Omega}$. Hence there exists a germ of $J$ holomorphic disc $\gamma$ such that $\gamma(0)=0$ and $\frac{\partial^{k} \gamma}{\partial x^{k}}(0)=D_{X}^{k-1,0} X(0)$ for all $k \in \mathbb{N}^{*}$. 
From this point, the problem of finding a $J$-holomorphic disc included in $M$ with prescribed derivatives is reduced to finding a sequence of vector fields $\left(X_{l}\right)_{l}$ in $T^{J} M$ such that the sequence $\left(D_{X_{l}}^{k-1,0} X_{l}(0)\right)_{k \in \mathbb{N}^{*}}$ does not depend on $l$ and is such that $X_{l}$ commutes at order $l$ at 0 . Indeed, Theorem 3.1 gives a $J$-holomorphic disc $\gamma$ such that for all $k$ and all $l, \frac{\partial^{k} \gamma}{\partial x^{k}}(0)=D_{X_{l}}^{k-1,0} X_{l}(0)$. Since $X_{l}$ commutes at order $l$, Theorem 1 of [1] implies that $\gamma$ is tangent to $M$ at 0 at order $l+1$, for all $l$. As both $M$ and $\gamma$ are real analytic, it follows that $\gamma$ is actually included in $M$.

Starting from a vector field $X$ which does not commute but which satisfies the assumptions of Theorem 1.4 or 1.6, we inductively construct such a sequence of vector fields by solving systems of linear equations (see Lemma 4.3 and Lemma 4.6).

The paper is organized as follows. In section 2, we prove Theorem 1.1. In Section 3, we show the existence of $J$-holomorphic disc with prescribed derivatives at any order. Finally, in Section 4, we prove Theorem 1.4 and 1.6 and give examples of applications of these theorems.

\section{A counter-example to a generalization of Freeman's Theorem}

In this section, in order to prove Theorem 1.1, we exhibit a complex structure $J$ and a pseudoconvex hypersurface $M$ in $\mathbb{R}^{6}$, both real analytic, such that there exists a vector $v$ which belongs to the kernel of the Levi form of $M$ at the point 0 but which is not the derivative at 0 of some $J$-holomorphic disc $\gamma: \mathbb{D} \rightarrow \mathbb{R}^{2 n}$ included in $M$, despite the fact that the kernel of the Levi form of $M$ is a subbundle of $T^{J} M$. We will prove that in fact, there exists no $J$-holomorphic disc $\gamma$ tangent to $M$ at 0 at order 5 (i.e. such that $\left.\varphi \circ \gamma(\zeta)=O\left(\zeta^{5}\right)\right)$

Proof of Theorem 1.1: Let $\varphi: \mathbb{R}^{6} \rightarrow \mathbb{R}$ be the map defined by $\varphi\left(x_{1}, y_{1}, x_{2}, y_{2}, x_{3}, y_{3}\right)=$ $y_{1}$, and let $M$ be the set $M=\left\{z \in \mathbb{R}^{6} \varphi(z)=0\right\}$.

We define the six following vector fields

$$
\begin{aligned}
L_{1} & =\frac{\partial}{\partial x_{1}}, & L_{2} & =\frac{\partial}{\partial y_{1}}, \\
L_{3} & =\frac{\partial}{\partial x_{2}}-\frac{1}{2} y_{3}^{2} \frac{\partial}{\partial x_{1}}, & L_{4} & =\frac{\partial}{\partial y_{2}}+\left(-2 y_{3} x_{3}+x_{2}\right) \frac{\partial}{\partial x_{1}}, \\
L_{5} & =\frac{\partial}{\partial x_{3}}-y_{2} y_{3} \frac{\partial}{\partial x_{1}}, & L_{6} & =\frac{\partial}{\partial y_{3}}+x_{3} \frac{\partial}{\partial x_{2}}-\left(\frac{x_{3} y_{3}^{2}}{2}+x_{3} y_{2}\right) \frac{\partial}{\partial x_{1}},
\end{aligned}
$$

and the complex structure $J$ they induce by setting

$$
J L_{1}=L_{2}, \quad J L_{3}=L_{4}, \quad J L_{5}=L_{6} .
$$

Therefore $J(0)$ is the standard complex structure $J_{0}$ and $T M$ is spanned over $\mathbb{R}$ by $L_{1}$, $L_{3}, L_{4}, L_{5}$ and $L_{6}$, while $T^{J} M$ is spanned over $\mathbb{C}$ by $L_{3}$ and $L_{5}$.

When we compute the Lie brackets of the $L_{i}$ 's belonging to the complex tangent bundle, 
we get:

$$
\begin{aligned}
& {\left[L_{3}, L_{4}\right]=L_{1},} \\
& {\left[L_{3}, L_{5}\right]=0, \quad\left[L_{4}, L_{5}\right]=y_{3} \frac{\partial}{\partial x_{1}},} \\
& {\left[L_{3}, L_{6}\right]=y_{3} \frac{\partial}{\partial x_{1}} \quad\left[L_{4}, L_{6}\right]=0, \quad\left[L_{5}, L_{6}\right]=L_{3} .}
\end{aligned}
$$

Therefore

$$
\begin{aligned}
\hat{\mathcal{L}}_{\varphi}\left(L_{3}, L_{5}\right) & =0 \\
\hat{\mathcal{L}}_{\varphi}\left(L_{3}, J L_{3}\right) & =d \varphi\left(L_{2}\right)=1, \\
\hat{\mathcal{L}}_{\varphi}\left(L_{5}, J L_{5}\right) & =d \varphi\left(L_{3}\right)=0
\end{aligned}
$$

which thus implies that $M$ is pseudoconvex and that $\operatorname{ker} \mathcal{L}=\operatorname{span}_{\mathbb{C}}\left\{L_{5}\right\}$; therefore $\operatorname{ker} \mathcal{L}$ is a subbundle of $T^{J} M$.

Now let us assume that there exists a $J$-holomorphic disc $\gamma: \mathbb{D} \rightarrow \mathbb{R}^{2 n}$ tangent to $M$ at order 5 and such that $\frac{\partial \gamma}{\partial x}(0)=L_{5}(0)$. Theorem 1 from [1] implies that there exists a vector field $X \in T^{J} M$ such that $X$ commutes at order 4 at 0 , i.e. such that all the Lie brackets of $X$ and $J X$ of length at most 4 vanish at 0 , and such that $X(0)=\frac{\partial \gamma}{\partial x}(0)=L_{5}(0)$. We prove that such a vector field does not exist.

Let us consider $X=a L_{3}+b L_{4}+c L_{5}+d L_{6}$ where $a, b, c$ and $d$ are real valued functions. Let us assume that $X$ commutes at order 4 and that $X(0)=L_{5}(0)$. Therefore we have $a(0)=b(0)=d(0)=0$ and $c(0)=1$. We compute $[X, J X]$ :

$$
\begin{aligned}
{[X, J X]=} & \left(a^{2}+b^{2}\right) L_{1}+\left(c^{2}+d^{2}\right) L_{3}+(-X(b)-J X(a)) L_{3}+(X(a)-J X(b)) L_{4} \\
& +(-X(d)-J X(c)) L_{5}+(X(c)-J X(d)) L_{6}
\end{aligned}
$$

and since $[X, J X](0)=0$, it follows that

$$
X(b)(0)+J X(a)(0)=-1 .
$$

We also get from the computation of $[X, J X]$ that

$$
d \varphi(J[X, J X])=a^{2}+b^{2},
$$

thus

$$
X \cdot X \cdot d \varphi(J[X, J X])=2\left(X(a)^{2}+X(b)^{2}+a X \cdot X(a)+b X \cdot X(b)\right)
$$

and

$$
J X \cdot J X \cdot d \varphi(J[X, J X])=2\left(J X(a)^{2}+J X(b)^{2}+a J X \cdot J X(a)+b J X \cdot J X(b)\right) .
$$

Using the commutativity of $X$ at 0 , we will show that $X \cdot X \cdot d \varphi(J[X, J X])(0)=J X$. $J X \cdot d \varphi(J[X, J X])(0)=0$ which is incompatible with (1).

We have

$$
X \cdot d \varphi(J[X, J X])=d \varphi((X . J)[X, J X])+d \varphi(J \cdot X \cdot[X, J X])+D^{2} \varphi(X, J[X, J X])
$$


and

$$
\begin{aligned}
X & \cdot X \cdot d \varphi(J[X, J X]) \\
= & d \varphi((X \cdot X \cdot J)[X, J X])+2 d \varphi((X . J) \cdot X \cdot[X, J X])+d \varphi(J(X \cdot X \cdot[X, J X])) \\
& +D^{2} \varphi(X \cdot X, J[X, J X])+D^{2} \varphi(X,(X \cdot J)[X, J X])+D^{2} \varphi(X, J(X \cdot[X, J X])) \\
& +D^{3} \varphi(X, X, J[X, J X]) .
\end{aligned}
$$

Now, $[X, J X]$ vanishes at 0 so $[X,[X, J X]](0)=X \cdot[X, J X](0)$ and since $X$ commutes at 0 at order $4, X \cdot[X, J X](0)=0$.

Then we get

$$
\begin{aligned}
{[X,[X,[X, J X]]](0) } & =X \cdot[X,[X, J X]](0) \\
& =X \cdot(X \cdot[X, J X])(0)-X \cdot([X, J X] \cdot X)(0) .
\end{aligned}
$$

Using again $[X, J X](0)=0$, we get $X \cdot([X, J X] \cdot X)(0)=(X \cdot[X, J X]) \cdot X(0)$ and since $X \cdot[X, J X](0)=0$, we get $X \cdot([X, J X] \cdot X)(0)=0$. Therefore $X \cdot(X \cdot[X, J X])(0)=$ $[X,[X,[X, J X]]](0)$ and since $X$ commutes at order 4 at $0, X \cdot(X \cdot[X, J X])(0)=0$, which gives $X \cdot(X \cdot d \varphi(J[X, J X]))(0)=0$, and so $X(b)(0)=0$.

Analogously, we also have $J X \cdot(J X \cdot d \varphi(J[X, J X]))(0)=0$ and so $J X(a)(0)=0$.

Now, just notice that (1) and $X(b)(0)=J X(a)(0)=0$ are incompatible. This gives that there is no $J$-holomorphic disc $\gamma$ tangent at order 5 at 0 to $M$ such that $\frac{\partial \gamma}{\partial x}(0)=L_{5}(0)$.

\section{Existence of $J$-HOLOMORPHIC DISCS WITH PRESCRIBED DERIVATIVES}

In this section, we show under an appropriate assumption, that there exists a $J$-holomorphic disc $\gamma$ with prescribed derivatives of any order. More precisely, we prove the following

Theorem 3.1. Let $J$ be a real analytic complex structure in a neighborhood of the origin of $\mathbb{R}^{2 n}$, and let $\left(x_{k}\right)_{k}$ be a sequence of vectors of $\mathbb{R}^{2 n}$ such that $\sum_{k=0}^{\infty} \frac{k+1}{k !}\left|x_{k}\right| \leq r$, where $r>0$ is sufficiently small.

Then there exists a J-holomorphic disc $\gamma: \mathbb{D} \rightarrow \mathbb{R}^{2 n}$ such that for all $k \in \mathbb{N}, \frac{\partial^{k} \gamma}{\partial x^{k}}(0)=x_{k}$.

Before proving Theorem 3.1, we rewrite the condition of $J$-holomorphicity of a disc when $J$ is close to the standard structure $J_{0}$. As in $\left[4, \gamma: \mathbb{D} \rightarrow \mathbb{R}^{2 n}\right.$ is a $J$-holomorphic disc if and only if

$$
\frac{\partial \gamma}{\partial \bar{\zeta}}-A_{J}(\gamma) \frac{\partial \bar{\gamma}}{\partial \bar{\zeta}}=0
$$

with $A_{J}(z)=\left(J_{0}+J(z)\right)^{-1}\left(J_{0}-J(z)\right) C, C$ being the $\mathbb{R}$-linear application which corresponds to the complex conjugation on $\mathbb{R}^{2 n}=\mathbb{C}^{n}$.

Let $T: C^{\omega}(\mathbb{D}) \rightarrow C^{\omega}(\mathbb{D})$ be the map defined by $T(u)(\zeta):=\int_{[0, \bar{\zeta}]} u(\zeta, \omega) d \omega$. The real analytic function $T(u)$ is a primitive of $u$ with respect to $\bar{\zeta}$, that is $\frac{\partial T(u)}{\partial \bar{\zeta}}=u$. Let also $\Phi_{J}: C^{\omega}(\mathbb{D}) \rightarrow C^{\omega}(\mathbb{D})$ be the map defined by $\Phi_{J}(u)=u-T\left(A_{J}(u) \frac{\partial \bar{u}}{\partial \bar{\zeta}}\right)$.

Then, a real analytic function $\gamma: \mathbb{D} \rightarrow \mathbb{R}^{2 n}$ is $J$-holomorphic if and only if $\Phi_{J}(\gamma)$ is holomorphic in the classical way. We can now prove Theorem 3.1 
Proof of Theorem 3.1: The principle of the proof is analogous to the case of finite sequences: we first introduce two well chosen Banach algebras: an algebra $\tilde{\Omega}$ of vector sequences, and an algebra $\Omega$ of real analytic functions on $\mathbb{D}$. We set

$$
\begin{aligned}
& \tilde{\Omega}=\left\{\left(x_{k}\right)_{k} \in\left(\mathbb{R}^{2 n}\right)^{\mathbb{N}},\left\|\left(x_{k}\right)_{k}\right\|_{\sim}:=\sum_{k=0}^{\infty} \frac{k+1}{k !}\left|x_{k}\right|<\infty\right\}, \\
& \Omega=\left\{f=\sum_{k, l} f_{k, l} \zeta^{k} \bar{\zeta}^{l}: \mathbb{D} \rightarrow \mathbb{R}^{2 n},\|f\|:=\sum_{k, l=0}^{\infty}(1+k+l+k l)\left|f_{k, l}\right|<\infty\right\} .
\end{aligned}
$$

We also introduce two applications

$$
\begin{aligned}
& \varphi_{1}:\left\{\begin{array}{ccc}
\tilde{\Omega} & \longrightarrow C^{\omega}(\mathbb{D}) \\
\left(x_{k}\right)_{k} & \longmapsto \sum_{k} \frac{x_{k}}{k !} \zeta^{k}
\end{array},\right. \\
& \varphi_{2}:\left\{\begin{array}{c}
\Omega \longrightarrow\left(\mathbb{R}^{2 n}\right)^{\mathbb{N}} \\
f \longmapsto\left(\frac{\partial^{k} f}{\partial x^{k}}(0)\right)_{k} .
\end{array}\right.
\end{aligned}
$$

We will prove the following facts:

(i) $\varphi_{1}$ is an isometry from $\tilde{\Omega}$ into $\Omega$,

(ii) $\varphi_{2}$ maps continuously $\Omega$ into $\tilde{\Omega}$,

(iii) $\Phi_{J}: \Omega \rightarrow \Omega$ is invertible in a neighborhood of 0 ,

(iv) $\varphi_{2} \circ \Phi_{J}^{-1} \circ \varphi_{1}: \tilde{\Omega} \rightarrow \Omega$ is also invertible in a neighborhood of 0 .

Then, the disc $\gamma=\Phi_{J}^{-1} \circ \varphi_{1} \circ\left(\varphi_{2} \circ \Phi_{J}^{-1} \circ \varphi_{1}\right)^{-1}\left(\left(x_{k}\right)_{k}\right)$ will be the $J$-holomorphic disc we are looking for.

We first check fact (ii): $\varphi\left(\left(x_{k}\right)_{k}\right)=\sum_{k} \frac{x_{k}}{k !} \zeta^{k}$ and so, for all $l \neq 0$, the coefficient of $\zeta^{k} \bar{\zeta}^{l}$ in $\varphi_{1}\left(\left(x_{k}\right)_{k}\right)$ vanishes. Therefore

$$
\left\|\varphi_{1}\left(\left(x_{k}\right)_{k}\right)\right\|=\sum_{k}(1+k) \frac{\left|x_{k}\right|}{k !}=\left\|\left(x_{k}\right)_{k}\right\|_{\sim}
$$

and $\varphi_{1}$ is an isometry from $\tilde{\Omega}$ into $\Omega$.

We now check fact (iii): writing $\zeta$ as $\zeta=x+i y$, we get for all $k$ and $l$ that $\zeta^{k} \bar{\zeta}^{l}=$ $x^{k+l}+y P_{k, l}(x, y)$ where $P_{k, l}$ is a polynomial in $x$ and $y$. Therefore

$$
\left.\frac{\partial^{n} \zeta^{k} \bar{\zeta}^{l}}{\partial x^{n}}\right|_{\zeta=0}=\left\{\begin{array}{ll}
(k+l) ! & \text { if } k+l=n \\
0 & \text { otherwise }
\end{array} .\right.
$$

So, for $f=\sum_{k, l} f_{k, l} \zeta^{k} \bar{\zeta}^{l}$, we have $\frac{\partial^{n} f}{\partial x^{n}}(0)=n ! \sum_{k+l=n} f_{k, l}$ and

$$
\varphi_{2}(f)=\left(n ! \sum_{k+l=n} f_{k, l}\right)_{n \in \mathbb{N}},
$$

from which it follows that

$$
\begin{aligned}
\left\|\varphi_{2}(f)\right\|_{\sim} & \leq \sum_{n}(n+1) \sum_{k+l=n}\left|f_{k, l}\right| \\
& \leq\|f\| .
\end{aligned}
$$


So $\varphi_{2}(f)$ belongs to $\tilde{\Omega}$ and $\varphi_{2}: \Omega \rightarrow \tilde{\Omega}$ is continuous.

In order to prove Fact (iii), we introduce for $f \in \Omega$ the functions $g=A_{J}(f) \frac{\partial \bar{f}}{\partial \bar{z}}$ and $G=T(g)$. Fact (iii) will be proved if we show that $G$ belongs to $\Omega$ and that $\|G\|<\frac{1}{2}\|f\|$ when $\|f\|$ is small, because $\Phi_{J}$ will then be an invertible perturbation of the identity in a neighborhood of $0 \in \Omega$. We will use the following lemma.

Lemma 3.2. Let $\mathcal{A}$ be the Banach algebra of complex valued functions defined by

$$
\mathcal{A}=\left\{u(z, \bar{z})=\sum_{k, l} u_{k, l} z^{k} \bar{z}^{l},\|f\|_{*}:=\sum_{k, l}(1+k)\left|f_{k, l}\right|<\infty\right\},
$$

and let $F$ be an analytic function bounded on $\left\{z \in \mathbb{C}^{k},|z|<R\right\}, R>0$. For $r>0$, we denote by $\mathcal{A}_{r}$ the set $\mathcal{A}_{r}=\left\{f \in \mathcal{A},\|f\|_{*}<r\right\}$.

Then, for all $r \in] 0, R[$, the following properties hold true:

(i) for all $f_{1}, \ldots, f_{k} \in \mathcal{A}_{r}, F\left(f_{1}, f_{2}, \ldots, f_{k}\right)$ belongs to $\mathcal{A}$,

(ii) $\Psi_{F}:\left\{\begin{array}{cl}\mathcal{A}_{r}^{k} & \longrightarrow \mathcal{A} \\ \left(f_{1}, \ldots, f_{k}\right) & \longmapsto \Psi_{F}\left(f_{1}, \ldots, f_{k}\right)=F\left(f_{1}, \ldots, f_{k}\right)\end{array}\right.$ is continuous,

(iii) for all $f_{1}, \ldots, f_{k} \in \mathcal{A}_{r},\left\|\Psi_{F}\left(f_{1}, \ldots, f_{k}\right)\right\|_{*} \leq\left(\frac{R}{R-r}\right)^{k}\|F\|_{\infty}$.

We admit this lemma for the moment and finish the proof of Theorem 3.1 . We denote by $R$ the radius of convergence of $A_{J}$, and we apply Lemma 3.2 to $A_{J}$. If $f$ belongs to $\Omega$, each of its components belongs to $\mathcal{A}$ and has norm smaller than $\|f\|$. Therefore, for all $f$ belonging to $\Omega_{\frac{R}{2}}:=\left\{g \in \Omega,\|g\|<\frac{R}{2}\right\}$, Lemma 3.2 implies that each coefficient in the matrix $A_{J}(f)$ belongs to $\mathcal{A}$ and have norm smaller that $\left\|A_{J}\right\|_{\infty}$. Moreover $\left\|\frac{\partial \bar{f}}{\partial \bar{z}}\right\|_{*}=$ $\sum_{k, l}(1+l) k\left|f_{k, l}\right| \leq\|f\|$, and since $\mathcal{A}$ is a Banach algebra, we have $\|g\|_{*}=\left\|A_{J}(f) \frac{\partial \bar{f}}{\partial \bar{z}}\right\|_{*} \lesssim$ $\left\|A_{J}\right\|_{\infty}\|f\|$, uniformly with respect to $f$ and $J$.

Now we notice that $T: \mathcal{A}^{2 n} \rightarrow \Omega$ is continuous because for $u=\sum_{k, l} u_{k, l} z^{k} \bar{z}^{l} \in \mathcal{A}$ we have

$$
\int_{[0, \bar{z}]} u(z, \omega) d \omega=\sum_{k, l} \frac{1}{l+1} u_{k, l} z^{k \bar{z}^{l+1}}
$$

and

$$
\begin{aligned}
\sum_{k, l} \frac{k+(l+1)+1+k(l+1)}{l+1}\left|u_{k, l}\right| & =\sum_{k, l} \frac{k+1}{l+1}\left|u_{k, l}\right|+(k+1)\left|u_{k, l}\right| \\
& \leq 2\|u\|_{*} .
\end{aligned}
$$

Therefore $G=T(g)$ belongs to $\Omega$ and $\|G\| \leq 2\|g\|_{*} \lesssim\left\|A_{J}\right\|_{\infty}\|f\|$. This proves that $\Phi_{J}(f)$ belongs to $\Omega$ for all $f \in \Omega_{\frac{R}{2}}$ and that $\Phi_{J}: \Omega_{\frac{R}{2}} \rightarrow \Omega$ is continuous. Moreover, provided $J$ is close enough to the standard structure, $\left\|A_{J}\right\|_{\infty}$ is arbitrarily small and so $\|G\| \leq \frac{1}{2}\|f\|$ which implies that $\Phi_{J}$ is a small perturbation of the identity. Thus $\Phi_{J}: \Omega_{\frac{R}{2}} \rightarrow \Phi_{J}\left(\Omega_{\frac{R}{2}}\right)$ is continuously invertible.

Now Fact (iv) is an immediate consequence of the previous facts because, since $\varphi_{2} \circ \varphi_{1}$ is the identity over $\tilde{\Omega}, \varphi_{2} \circ \Phi_{J}^{-1} \circ \varphi_{1}$ is in fact a continuous perturbation of the identity which is arbitrarily small, provided $J$ is close enough to the standard structure.

Now, for any sequence $\left(x_{k}\right)_{k}$ of vectors of $\mathbb{R}^{2 n}$, provided $\left\|\left(x_{k}\right)_{k}\right\|<\frac{R}{2}, \gamma=\Phi_{J}^{-1} \circ \varphi_{1} \circ$ 
$\left(\varphi_{2} \circ \Phi_{J}^{-1} \circ \varphi_{1}\right)^{-1}\left(\left(x_{k}\right)_{k}\right)$ is a $J$-holomorphic disc such that $\varphi_{2}(\gamma)=\left(x_{k}\right)_{k}$. To conclude the proof of the theorem, we have to prove the crucial Lemma 3.2 .

Proof of Lemma 3.2: The proof of this Lemma is inspired from abstract harmonic analysis and more precisely from Theorem $24 \mathrm{D}$ of [6]. In order to make the proof clearer, we prove the lemma for $k=2$, that is for $F: \mathbb{C}^{2} \rightarrow \mathbb{C}$, analytic over $P(0, R)=\{(z, w) \in$ $\left.\mathbb{C}^{2},|z|^{2}+|w|^{2}<R^{2}\right\}, R>0$. The case $k>2$ is a direct generalization of the case $k=2$.

Let $f=\sum_{k, l} f_{k, l} \zeta^{k} \bar{\zeta}^{l}$ and $g=\sum_{k, l} g_{k, l} \zeta^{k} \bar{\zeta}^{l}$ belonging to $\mathcal{A}$ be such that $\|f\|_{*}<r$ and $\|g\|_{*}<r$ for some $\left.r \in\right] 0, R\left[\right.$. We write $F(z, w)=\sum_{k, l} F_{k, l} z^{k} w^{l}$ and $F(f, g)(\zeta)=$ $\sum_{k, l} h_{k, l} \zeta^{k} \bar{\zeta}^{l}$. In order to determine a suitable expression of $h_{k, l}$ we introduce the Banach algebra

$$
\hat{\mathcal{A}}=\left\{\left(u_{k, l}\right)_{k, l} \subset \mathbb{R}^{2 n},\left\|\left(u_{k, l}\right)_{k, l}\right\|_{\hat{*}}:=\sum_{k, l}(1+k)\left|u_{k, l}\right|<\infty\right\}
$$

that we trivially identify to $\mathcal{A}$ via the application

$$
\phi:\left\{\begin{array}{cll}
\mathcal{A} & \longrightarrow \hat{\mathcal{A}} \\
\sum_{k, l} u_{k, l} \zeta^{k} \bar{\zeta}^{l} & \longmapsto & \left(u_{k, l}\right)_{k, l}
\end{array} .\right.
$$

We denote by $\mathfrak{e}$ the unit of $\hat{\mathcal{A}}, \mathfrak{e}_{k, l}=1$ if $k=l=0$, and 0 otherwise and we denote by $\mathbf{1}$ the constant function which equals 1 so that $\phi(\mathbf{1})=\mathfrak{e}$. We have

$$
h_{k, l}=\sum_{n, m} F_{n, m} \phi\left(f^{n} g^{m}\right)_{k, l}
$$

and using Cauchy's Formula we get:

$$
h_{k, l}=\sum_{n, m} \frac{1}{(2 i \pi)^{2}} \int_{\substack{|\lambda|=R \\|\mu|=R}} \frac{F(\lambda, \mu)}{\lambda^{n+1} \mu^{m+1}} \phi\left(f^{n} g^{m}\right)_{k, l} d \lambda d \mu .
$$

Since $\|f\|_{*}<r$ and $\|g\|_{*}<r$, we have $\left\|f^{n} g^{m}\right\|_{*}<r^{n+m}$ so $\left|\phi\left(f^{n} g^{m}\right)_{k, l}\right|<r^{n+m}$. Therefore if $r<R$, the series $\sum_{n, m} \frac{1}{\lambda^{n} \mu^{m}} \phi\left(f^{n}, g^{m}\right)_{k, l}$ converges normally for all $\lambda$ and $\mu$ in $\mathbb{C}$ such that $|\lambda|=|\mu|=R$, and we can exchange the signs $\int$ and $\sum$ in the last expression of $h_{k, l}$ and which gives

$$
h_{k, l}=\frac{1}{(2 i \pi)^{2}} \int_{\substack{|\lambda|=R \\|\mu|=R}} F(\lambda, \mu) \sum_{n, m}\left(\phi\left(\frac{f^{n}}{\lambda^{n+1}}\right) \phi\left(\frac{g^{m}}{\mu^{m+1}}\right)\right)_{k, l} d \lambda d \mu .
$$

Since $\|f\|_{*}<r<|\lambda|$ and $\|g\|_{*}<r<|\mu|$, we have $(\lambda \mathfrak{e}-\phi(f))^{-1}=\sum_{n} \phi\left(\frac{f^{n}}{\lambda^{n+1}}\right)$ and $(\mu \mathfrak{e}-\phi(g))^{-1}=\sum_{m} \phi\left(\frac{g^{m}}{\mu^{m+1}}\right)$ which yields

$$
h_{k, l}=\frac{1}{(2 i \pi)^{2}} \int_{\substack{|\lambda|=R \\|\mu|=R}} F(\lambda, \mu)\left((\lambda \mathfrak{e}-\phi(f))^{-1}(\mu \mathfrak{e}-\phi(g))^{-1}\right)_{k, l} d \lambda d \mu .
$$


Using this expression of $h_{k, l}$, it follows that

$$
\begin{aligned}
& \sum_{k, l}(k+1)\left|h_{k, l}\right| \\
& \leq \frac{R^{2}}{(2 \pi)^{2}} \int_{0}^{2 \pi} \int_{0}^{2 \pi}\left|F\left(R e^{i \theta}, R e^{i \varphi}\right)\right| \sum_{k, l}(k+1)\left|\left(\left(R e^{i \theta} \mathfrak{e}-\phi(f)\right)^{-1}\left(R e^{i \varphi} \mathfrak{e}-\phi(g)\right)^{-1}\right)_{k, l}\right| d \theta d \varphi \\
& \leq \frac{R^{2}}{(2 \pi)^{2}} \int_{0}^{2 \pi} \int_{0}^{2 \pi}\left|F\left(R e^{i \theta}, R e^{i \varphi}\right)\right|\left\|\left(R e^{i \theta} \mathfrak{e}-\phi(f)\right)^{-1}\left(R e^{i \varphi} \mathfrak{e}-\phi(g)\right)^{-1}\right\|_{\hat{*}} d \theta d \varphi .
\end{aligned}
$$

Now, using again the identity $(\lambda \mathfrak{e}-\phi(f))^{-1}=\sum_{n} \phi\left(\frac{f^{n}}{\lambda^{n+1}}\right)$, for $\lambda=R e^{i \theta}$ we get

$$
\left\|(\lambda \mathfrak{e}-\phi(f))^{-1}\right\|_{\hat{*}} \leq \frac{1}{R-r} .
$$

The same holds true for $g$ and so $\|F(f, g)\|_{*}=\sum_{k, l}(k+1)\left|h_{k, l}\right| \leq\left(\frac{R}{R-r}\right)^{2}\|F\|_{\infty}$, which implies that $F(f, g)$ belongs to $\mathcal{A}$ and that (iii) of Lemma 3.2 holds true.

In order to establish the continuity of $\Psi_{F}$, let $\tilde{f}$ and $\tilde{g}$ be two functions of $\mathcal{A}$ such that $\|\tilde{f}\|_{*}<r$ and $\|\tilde{g}\|_{*}<r$ and set $F(\tilde{f}, \tilde{g})=\sum_{k, l} \tilde{h}_{k, l} \zeta^{k} \bar{\zeta}^{l}$. We have

$h_{k, l}-\tilde{h}_{k, l}=$

$\frac{1}{(2 i \pi)^{2}} \int_{\substack{|\lambda|=R \\|\mu|=R}} F(\lambda, \mu)\left((\lambda \mathfrak{e}-\phi(f))^{-1}(\mu \mathfrak{e}-\phi(g))^{-1}-(\lambda \mathfrak{e}-\phi(\tilde{f}))^{-1}(\mu \mathfrak{e}-\phi(\tilde{g}))^{-1}\right)_{k, l} d \lambda d \mu$.

This yields

$$
\begin{aligned}
\|F(f, g)-F(\tilde{f}, \tilde{g})\|_{*} \leq & \frac{R^{2}}{4 \pi^{2}} \int_{0}^{2 \pi} \int_{0}^{2 \pi}\left|F\left(\operatorname{Re}^{i \theta}, R e^{i \varphi}\right)\right| \cdot \|\left(\operatorname{Re}^{i \theta} \mathfrak{e}-\phi(f)\right)^{-1}\left(\operatorname{Re}^{i \varphi} \mathfrak{e}-\phi(g)\right)^{-1} \\
& -\left(\operatorname{Re} e^{i \theta} \mathfrak{e}-\phi(\tilde{f})\right)^{-1}\left(R e^{i \varphi} \mathfrak{e}-\phi(\tilde{g})\right)^{-1} \|_{\tilde{*}} d \theta d \varphi .
\end{aligned}
$$

We have the following inequality:

$$
\begin{aligned}
\|\left(R e^{i \theta} \mathfrak{e}-\phi(f)\right)^{-1} & -\left(R e^{i \varphi} \mathfrak{e}-\phi(\tilde{f})\right)^{-1} \| \\
& \leq\left\|\left(R e^{i \theta} \mathfrak{e}-\phi(f)\right)^{-1} \cdot\left(R e^{i \varphi} \mathfrak{e}-\phi(\tilde{f})\right)^{-1}\right\| \cdot\|\phi(f)-\phi(\tilde{f})\|_{\hat{*}} \\
& \leq \frac{1}{(R-r)^{2}}\|f-\tilde{f}\|_{*} .
\end{aligned}
$$

Using this estimate of $\left\|\left(R e^{i \theta} \mathfrak{e}-\phi(f)\right)^{-1}-\left(R e^{i \theta} \mathfrak{e}-\phi(\tilde{f})\right)^{-1}\right\|$ and the corresponding one for $\left\|\left(R e^{i \varphi} \mathfrak{e}-\phi(g)\right)^{-1}-\left(R e^{i \varphi} \mathfrak{e}-\phi(\tilde{g})\right)^{-1}\right\|$, we get

$$
\|F(f, g)-F(\tilde{f}, \tilde{g})\|_{*} \leq \frac{R^{2}}{(R-r)^{3}}\|F\|_{\infty} \cdot\left(\|f-\tilde{f}\|_{*}+\|g-\tilde{g}\|_{*}\right)
$$

which proves that $\Psi_{F}$ is continuous.

Under a simple growth condition of a sequence $\left(x_{k}\right)_{k}$, the following corollary gives the existence of a germ of $J$-holomorphic disc $\gamma$ such that for all $k \in \mathbb{N}, \frac{\partial^{k} \gamma}{\partial x^{k}}(0)=x_{k}$. 
Corollary 3.3. Let $J$ be a real analytic complex structure in a neighborhood of the origin of $\mathbb{R}^{2 n}$, and let $\left(x_{k}\right)_{k}$ be a sequence of vectors of $\mathbb{R}^{2 n}$. Assume that $x_{0}$ is close enough to the origin and that there exists $R>0$ such that for all $k \in \mathbb{N}^{*},\left|x_{k}\right| \leq k ! R^{k}$.

Then there exists a germ of J-holomorphic disc $\gamma$ such that for all $k \in \mathbb{N}, \frac{\partial^{k} \gamma}{\partial x^{k}}(0)=x_{k}$.

Proof: Let $r>0$ be the constant given by Theorem 3.1 and for $\alpha>0$ let $\left(\tilde{x}_{k}\right)_{k}$ be the sequence defined by $\tilde{x}_{k}=\left(\frac{\alpha}{R}\right)^{k} x_{k}$.

Then $\sum_{k=1}^{\infty} \frac{k+1}{k !}\left|\tilde{x}_{k}\right| \leq \sum_{k=1}^{\infty}(k+1) \alpha^{k}$, so if $\left|x_{0}\right|<r$ and if $\alpha$ is small enough, we have $\sum_{k=0}^{\infty} \frac{k+1}{k !}\left|\tilde{x}_{k}\right|<r$. Therefore we can apply Theorem 3.1 to $\left(\tilde{x}_{k}\right)_{k}$ and so there exists a $J$-holomorphic disc $\tilde{\gamma}: \mathbb{D} \rightarrow \mathbb{R}^{2 n}$ such that $\frac{\partial^{k} \tilde{\gamma}}{\partial x^{k}}(0)=\tilde{x}_{k}$ for all $k$.

Now setting $\gamma(\zeta)=\tilde{\gamma}\left(\frac{R}{\alpha} \zeta\right)$ for $\zeta \in \mathbb{C}$ such that $|\zeta|<\frac{\alpha}{R}$, we get a germ of $J$-holomorphic disc such that $\frac{\partial^{k} \gamma}{\partial x^{k}}(0)=x_{k}$ for all $k$.

Given a point $p$ in $M$ and an analytic vector field $X$, we aim at finding a germ of $J$-holomorphic disc $\gamma$ such that $\gamma(0)=p$ and $\frac{\partial^{k} \gamma}{\partial x^{k}}(0)=D_{X}^{k-1,0} X(p)$ for $k \in \mathbb{N}^{*}$ by applying Corollary 3.3 to the sequence $\left(x_{k}\right)_{k}$ defined by $x_{0}=p$ and $x_{k}=D_{X}^{k-1,0} X(p), k \in \mathbb{N}^{*}$. For this, we have to prove that the sequence $\left(D_{X}^{k-1,0} X(p)\right)_{k}$ satisfies the assumption of this corollary. The estimates needed will be given in the next proposition, but before we state it, we need the following notation.

If $X$ is a real analytic vector field in the polydisc $\mathcal{P}(0, R)=\left\{\left(x_{1}, y_{1}, \ldots, x_{n}, y_{n}\right) \in\right.$ $\left.\mathbb{R}^{2 n},\left|x_{i}\right|<R,\left|y_{i}\right|<R, i=1, \ldots, n\right\}$, we denote by $\tilde{X}$ the "polarization" of $X$. More precisely, if $X(z, \bar{z})=\sum_{\nu, \mu} a_{\nu, \mu} z^{\nu} \bar{z}^{\mu}$ where $\nu$ and $\mu$ are multi-indices of $\mathbb{N}^{n}, z=x+i y$ belongs to $\mathbb{C}^{n}$ and $a_{\nu, \mu}$ belongs to $\mathbb{R}^{2 n}$, we set $\tilde{X}(z, \zeta)=\sum_{\nu, \mu} a_{\nu, \mu} z^{\nu} \zeta^{\mu}$ which is defined for all $z$ and $\zeta$ such that $\left|z_{j}\right|,\left|\zeta_{j}\right|<R, j=1, \ldots, n$. We then set

$$
c_{R}(X)=\sup _{\left|z_{j}\right|,\left|\zeta_{j}\right|<R}|\tilde{X}(z, \zeta)| .
$$

We can now state the estimates of $D_{X}^{k, 0} X(0)$ :

Proposition 3.4. Let $X$ be an analytic vector field in a neighborhood of the origin of $\mathbb{R}^{2 n}$. Then for all $R>0$ small enough and for all $k$

$$
\left|D_{X}^{k, 0} X(0)\right| \leq 2 n k !\left(\frac{8 n c_{R}(X)}{R}\right)^{k} .
$$

The proof of Proposition 3.4 relies on the following combinatorial Lemmata.

Lemma 3.5. For all $\alpha_{1}, \ldots, \alpha_{k} \in \mathbb{N}$ such that $\alpha_{1}+\ldots+\alpha_{k}=k$, we have

$$
\alpha_{1} ! \ldots \alpha_{k} ! \leq k ! \text {. }
$$

Proof: Without restriction we assume that $\alpha_{1}, \ldots, \alpha_{r} \geq 1$ and $\alpha_{r+1}=\ldots=\alpha_{k}=0, r \leq k$ so that $\alpha_{1}+\ldots+\alpha_{r}=k$.

The number $k$ ! is the number of permutations of the set $\{1, \ldots, k\}$ whereas $\alpha_{1} ! \ldots \alpha_{r}$ ! is the number of permutations of $\{1, \ldots, k\}$ which leave stable each of the sets $\left\{1, \ldots, \alpha_{1}\right\}$, $\left\{\alpha_{1}+1, \ldots, \alpha_{1}+\alpha_{2}\right\}, \ldots,\left\{\alpha_{1}+\ldots+\alpha_{r-1}+1, \ldots, k\right\}$. Therefore $\alpha_{1} ! \ldots \alpha_{k} ! \leq k !$

Lemma 3.6. There are exactly $\left(\begin{array}{c}2 k-1 \\ k\end{array}\right)$ distinct $k$-tuples $\left(\alpha_{1}, \ldots, \alpha_{k}\right) \in \mathbb{N}^{k}$ such that $\alpha_{1}+$ $\ldots+\alpha_{k}=k$. 
Proof: To a $k$-tuple $\left(\alpha_{1}, \ldots, \alpha_{k}\right) \in \mathbb{N}^{k}$ we associate the $k$-tuple $\left(\beta_{1}, \ldots, \beta_{k}\right)=\left(\alpha_{1}+\right.$ $\left.1, \ldots, \alpha_{k}+1\right)$ so that $\beta_{1}+\ldots+\beta_{k}=2 k$. This correspondence is a bijection, and now we count the number of such $k$-tuples $\left(\beta_{1}, \ldots, \beta_{k}\right)$.

The sum $1+1+\ldots+1$ ( $2 k$-times) equals $2 k$ and there are as many ways of writing $\beta_{1}+\ldots+\beta_{k}=2 k$ as ways of separating the number 1 in the sum $1+1+\ldots+1=2 k$ with $k-1$-sticks, that is $\left(\begin{array}{c}2 k-1 \\ k-1\end{array}\right)$ ways.

Lemma 3.7. For all $k \in \mathbb{N}$ we have

$$
\left(\begin{array}{c}
2 k-1 \\
k-1
\end{array}\right) \leq \frac{4^{k}}{\sqrt{\pi k}}
$$

Proof: This is a simple consequence of Stirling's formula.

We now prove Proposition 3.4.

Proof of Proposition 3.4: Let $X$ be a real analytic vector field in a neighborhood of the origin in $\mathbb{R}^{2 n}$. Let $R>0$ be such that $X$ is defined and continuous on $\overline{P(0, R)}$.

We write $X$ in local coordinates as $X=\sum_{j=1}^{2 n} X_{j} \frac{\partial}{\partial x_{j}}$. In order to establish the proposition, we need an upper bound for the quantity:

$$
X_{i_{1}} \frac{\partial}{\partial x_{i_{1}}}\left(X_{i_{2}} \frac{\partial}{\partial x_{i_{2}}}\left(\ldots\left(X_{i_{k-1}} \frac{\partial X_{i_{k}}}{\partial x_{i_{k-1}}}\right) \ldots\right)(0) .\right.
$$

In the term above, we denote by $j_{i}$ the cardinal of the set $\left\{l, i_{l}=i\right\}$.

In (2), for $i \in\{1, \ldots, 2 n\}$ and for $\alpha_{j}^{(i)}, j \in\{1, \ldots, k\}$, such that $\sum_{j} \alpha_{j}^{(i)}=j_{i}$, the term $\prod_{j=1}^{k} \frac{\partial^{\alpha_{j}^{(1)}+\ldots+\alpha_{j}^{(2 n)}} X_{i_{j}}}{\partial_{x_{j}^{(1)}}^{\alpha^{(1)}} \ldots x_{2 n}^{\alpha_{j}^{(2 n)}}}(0)$ appears less than $\left({ }_{\left(\alpha_{1}^{(1)}, \ldots, \alpha_{k}^{(1)}\right)}^{j_{1}}\right) \ldots\left({\left(\alpha_{1}^{(2 n)}, \ldots, \alpha_{k}^{(2 n)}\right)}_{j_{2 n}}\right)$ times.

On the other hand, from Cauchy's inequalities, we get

$$
\left|\frac{\partial^{\alpha_{j}^{(1)}+\ldots+\alpha_{j}^{(2 n)}} X_{i_{j}}}{\partial x_{1}^{\alpha_{j}^{(1)}} \ldots \partial x_{2 n}^{\alpha_{j}^{(2 n)}}}(0)\right| \leq \alpha_{j}^{(1)} ! \ldots \alpha_{j}^{(2 n)} ! \frac{c_{R}(X)}{R^{\alpha_{j}^{(1)}+\ldots+\alpha_{j}^{(2 n)}}} .
$$

This yields

$$
\mid X_{i_{1}} \frac{\partial}{\partial x_{i_{1}}}\left(X_{i_{2}} \frac{\partial}{\partial x_{i_{2}}}\left(\ldots\left(X_{i_{k-1}} \frac{\partial X_{i_{k}}}{\partial x_{i_{k-1}}}\right) \ldots\right)(0) \mid \leq \sum_{\substack{\alpha_{1}^{(1)}+\ldots+\alpha_{k}^{(1)}=j_{1} \\ \vdots \\ \alpha_{1}^{(2 n)}+\ldots+\alpha_{k}^{(2 n)}=j_{2 n}}} j_{1} ! \ldots j_{2 n} !\left(\frac{c_{R}(X)}{R}\right)^{k} .\right.
$$

Applying successively Lemma 3.6, Lemma 3.7 and Lemma 3.5 we get

$$
\begin{aligned}
\mid X_{i_{1}} \frac{\partial}{\partial x_{i_{1}}}\left(X_{i_{2}} \frac{\partial}{\partial x_{i_{2}}}\left(\ldots\left(X_{i_{k-1}} \frac{\partial X_{i_{k}}}{\partial x_{i_{k-1}}}\right) \ldots\right)(0) \mid\right. & \leq 4^{j_{1}+\ldots+j_{2 n}} j_{1} ! \ldots j_{2 n} !\left(\frac{c_{R}(X)}{R}\right)^{k} \\
& \leq k !\left(\frac{4 c_{R}(X)}{R}\right)^{k} .
\end{aligned}
$$

Now, since there are at most $(2 n)^{k}$ terms $X_{i_{1}} \frac{\partial}{\partial x_{i_{1}}}\left(X_{i_{2}} \frac{\partial}{\partial x_{i_{2}}}\left(\ldots\left(X_{i_{k-1}} \frac{\partial X_{i_{k}}}{\partial x_{i_{k-1}}}\right) \ldots\right)(0)\right.$ in $D_{X}^{k, 0} X(0)$,

$$
\left|D_{X}^{k, 0} X(0)\right| \leq 2 n k !\left(\frac{8 n c_{R}(X)}{R}\right)^{k}
$$


which proves the claim.

\section{J-HOLOMORPHIC DISCS AND HYPERSURFACES}

In this section, given a point $p \in M$ close enough to the origin and a vector $v \in T_{p}^{J} M$, we look for a $J$-holomorphic disc $\gamma$ included in $M$ such that $\frac{\partial \gamma}{\partial x}(p)=v$. Our strategy will be the following: we will look for a sequence of vector fields $\left(X_{k}\right)_{k \in \mathbb{N}^{*}}$ such that

(i) For all $k$, the vector field $X_{k}$ belongs to $T^{J} M$,

(ii) $X_{1}(p)=v$,

(iii) $D_{X_{k}}^{l, 0} X_{k}(p)=D_{X_{1}}^{l, 0} X_{1}(p)$ for all $k$ in $\mathbb{N}^{*}$ and $l$ in $\mathbb{N}$,

(iv) $X_{k}$ commutes at order $k$ at $p$.

Then, applying Proposition 3.4 to $X_{1}$ and corollary 3.3 to the sequence $\left(x_{k}\right)_{k}$ defined by $x_{0}=p$ and $x_{k}=D_{X_{1}}^{k-1,0} X_{1}(p)$ for $k \in \mathbb{N}^{*}$, we will get a germ of $J$-holomorphic disc $\gamma$ such that $\gamma(0)=p$ and $\frac{\partial^{k} \gamma}{\partial x^{k}}(p)=D_{X_{1}}^{k-1,0} X_{1}(p)$ for all $k \in \mathbb{N}^{*}$, and in particular $\frac{\partial \gamma}{\partial x}(p)=v$. In order to check that $\gamma$ is included in $M$, we will use Theorem 1 of [1]: since $X_{k}$ commutes at order $k$ at $p$, and since $\frac{\partial^{l} \gamma}{\partial x^{l}}(0)=D_{X_{k}}^{l-1,0} X_{k}(p)$ for all $l \leq k, \gamma$ is tangent to $M$ at $p$ at order $k+1$. Now, since this holds for all $k$, this implies that $\gamma$ is tangent to $M$ at $p$ at any order. As $M$ and $\gamma$ are real analytic, $\gamma$ is in fact included in $M$. In order to construct such a sequence of vector fields, we will need the following propositions.

Proposition 4.1. Let $X$ be any real analytic vector field in a neighborhood of the origin. Then the following assertions are equivalent:

(i) For all $0 \leq k \leq k_{0}-2$ and all $X_{1}, \ldots, X_{k} \in\{X, J X\}, X_{1} \cdot X_{2} \ldots X_{k} \cdot[X, J X](0)=0$,

(ii) $X$ commutes at order $k_{0}$ at 0 .

Moreover, in that case, for all integers $k$ and $l$ such that $0 \leq l \leq k \leq k_{0}-1$ the following holds:

$$
X_{1} \ldots X_{l} \cdot\left[X_{l+1},\left[\ldots\left[X_{k},[X, J X]\right] \ldots\right](0)=\left[X_{1},\left[X_{2}, \ldots\left[X_{k},[X, J X]\right] \ldots\right](0) .\right.\right.
$$

Remark 1. We point out that (3) holds true for $0 \leq k \leq k_{0}-1$ even if (ii) is true only for $0 \leq k \leq k_{0}-2$.

Proof of proposition 4.1: The equivalence of (ii) and (iii) was shown in [1], proposition 13. The second assertion is proved by induction on $l$, the case $l=0$ being trivial.

We assume that the identity holds true for $l \leq k \leq k_{0}-2$ and we denote by $Y$ the vector field $Y=\left[X_{l+2},\left[\ldots,\left[X_{k},[X, J X]\right] \ldots\right]\right]$. Then, since $\tilde{X}_{1} \ldots \tilde{X}_{l} \cdot Y(0)=0$ for all $\tilde{X}_{1}, \ldots \tilde{X}_{l} \in\{X, J X\}$, we have

$$
\begin{aligned}
& X_{1} \ldots X_{l}\left[X_{l+1},\left[X_{l+2}, \ldots\left[X_{k},[X, J X]\right] \ldots\right](0)\right. \\
& =X_{1} \ldots X_{l} \cdot X_{l+1} \cdot Y(0)-X_{1} \ldots X_{l} \cdot Y \cdot X_{l+1}(0) \\
& =X_{1} \ldots X_{l} \cdot X_{l+1} \cdot Y(0) .
\end{aligned}
$$


Proposition 4.2. Let $X$ be a vector field commuting at order $k$ at $0, k \geq 2$. Then for all $X_{1}, \ldots, X_{k} \in\{X, J X\}$, we have

$$
\left[X_{1},\left[\ldots,\left[X_{k},[X, J X]\right]\right]\right](0)=\underbrace{[J X,[\ldots,[J X}_{q}, \underbrace{[X,[\ldots,[X}_{p},[X, J X] \ldots](0)
$$

where $p=\#\left\{i, X_{i}=X\right\}$ and $q=\#\left\{i, X_{i}=J X\right\}$.

Proof: It suffices to show that for all $l \leq k-2$ we have

$$
\begin{aligned}
{\left[X_{1},\left[\ldots,\left[X_{l},\left[X,\left[J X,\left[X_{l+3},[\ldots,[X, J X] \ldots](0)\right.\right.\right.\right.\right.\right.} & \\
& =\left[X_{1},\left[\ldots,\left[X_{l},\left[J X,\left[X,\left[X_{l+3},[\ldots,[X, J X] \ldots](0) .\right.\right.\right.\right.\right.\right.
\end{aligned}
$$

We set $Y=\left[X_{l+3},[\ldots,[X, J X] \ldots]\right.$. From Jacobi identity we have:

$$
[X,[J X, Y]]=[J X,[X, Y]]+[[X, J X], Y] .
$$

From Proposition 4.1, for all $0 \leq \tilde{l} \leq l$ and all $\tilde{X}_{1}, \ldots, \tilde{X}_{\tilde{l}} \in\{X, J X\}$, we have $\tilde{X}_{1} \ldots \tilde{X}_{\tilde{l}}$. $[X, J X](0)=0$ which yields

$$
\left[X_{1}, \ldots,\left[X_{l},[X, J X] \cdot Y\right] \ldots\right](0)=0
$$

Analogously, it follows from Proposition 4.1 that $\left[X_{1}, \ldots,\left[X_{l}, Y \cdot[X, J X]\right] \ldots\right](0)=0$ which with (5) gives

$$
\left[X_{1}, \ldots,\left[X_{l},[[X, J X], Y]\right] \ldots\right](0)=0
$$

and with (4) we end the proof of the proposition.

Taking our strategy into account, the proof of Theorem 1.4 will be a direct consequence of the following lemma:

Lemma 4.3. Let $X \in T^{J} M$ be a real analytic vector field in a neighborhood of $0 \in M$ such that $\mathbb{L}(X)$, the Lie algebra generated by $X$, is included in $T^{J} M$.

Then there exists a sequence $\left(X_{k}\right)_{k \in \mathbb{N}^{*}}$ of vector fields in $T^{J} M$ such that

(a) $D_{X_{k}}^{l, 0} X_{k}(0)=D_{X}^{l, 0} X(0)$ for all $k$ in $\mathbb{N}^{*}$ and $l$ in $\mathbb{N}$,

(b) for all $k \in \mathbb{N}^{*}, X_{k}$ commutes at order $k$ at 0 .

Proof: We set $X_{1}=X$ and by induction on $k$ we construct a sequence $\left(X_{k}\right)_{k}$ of vector fields which satisfy (a) and (b) and which are such that for all $k, X_{k}$ belongs to $\mathbb{L}(X)$. Assuming $X_{k}$ constructed, we set

$$
Y_{p, q}=\underbrace{\left[J X_{k},\left[\ldots,\left[J X_{k}\right.\right.\right.}_{q}, \underbrace{\left[X_{k},\left[\ldots,\left[X_{k}\right.\right.\right.}_{p}\left[X_{k}, J X_{k}\right] \ldots]
$$

for all $p$ and $q$ such that $p+q=k-1$, and we look for $X_{k+1}$ as

$$
X_{k+1}=X_{k}+\sum_{p+q=k-1}\left(a_{p, q}+b_{p, q} J\right) Y_{p, q},
$$

where $a_{p, q}$ and $b_{p, q}$ are real analytic functions which vanish at order $k-1$ at the origin. Hence $X_{k+1}$ already belongs to $\mathbb{L}(X)$ and we have to show that $a_{p, q}$ and $b_{p, q}$ can be chosen 
so that (a) and (b) hold. We compute $\left[X_{k+1}, J X_{k+1}\right]$ :

$$
\begin{aligned}
& {\left[X_{k+1}, J X_{k+1}\right]=} \\
& =\left[X_{k}, J X_{k}\right]+\sum_{p+q=k-1}\left(\left(X_{k}\left(a_{p, q}\right)-J X_{k}\left(b_{p, q}\right)\right) J Y_{p, q}+\left(-X_{k}\left(b_{p, q}\right)-J X_{k}\left(a_{p, q}\right)\right) Y_{p, q}\right) \\
& +\sum_{p+q=k-1}\left(a_{p, q}^{2}+b_{p, q}^{2}\right)\left[Y_{p, q}, J Y_{p, q}\right] \\
& +\sum_{p+q=k-1}\left(a_{p, q}\left(\left[X_{k}, J Y_{p, q}\right]+\left[Y_{p, q}, J X_{k}\right]\right)-b_{p, q}\left(\left[X_{k}, Y_{p, q}\right]+\left[J X_{k}, J Y_{p, q}\right]\right)\right) \\
& +\sum_{\substack{p+q=k-1 \\
r+s=k-1}}\left(a_{r, s} Y_{r, s}\left(a_{p, q}\right)+b_{r, s} J Y_{r, s}\left(a_{p, q}\right)-a_{r, s} J Y_{r, s}\left(b_{p, q}\right)+b_{r, s} Y_{r, s}\left(b_{p, q}\right)\right) J Y_{p, q} \\
& +\sum_{\substack{p+q=k-1 \\
r+s=k-1}}\left(-a_{r, s} Y_{r, s}\left(b_{p, q}\right)-b_{r, s} J Y_{r, s}\left(b_{p, q}\right)-a_{r, s} J Y_{r, s}\left(a_{p, q}\right)+b_{r, s} Y_{r, s}\left(a_{p, q}\right)\right) Y_{p, q} .
\end{aligned}
$$

Since $a_{p, q}$ and $b_{p, q}$ vanish at order $k-1$ at the origin, and since $X_{k}$ commutes at order $k$ at 0 , for all $X^{(1)}, \ldots, X^{(k-2)} \in\left\{X_{k+1}, J X_{k+1}\right\}$ we have

$$
X^{(1)} \ldots X^{(k-2)}\left[X_{k+1}, J X_{k+1}\right](0)=0 .
$$

It then follows from Proposition 4.1 that $X_{k+1}$ already commutes at order $k$ at 0 . Moreover, $D_{X_{k+1}}^{r, 0} X_{k+1}(0)=D_{X_{k}}^{r, 0} X_{k}(0)$ for all $r \leq k-1$ and for all choices of functions $a_{p, q}$ and $b_{p, q}$, vanishing at order $k-1$ at 0 .

We now choose these functions so that $X_{k+1}$ commutes at order $k+1$ at the origin and so that $D_{X_{k+1}}^{r, 0} X_{k+1}(0)=D_{X_{k}}^{r, 0} X_{k}(0)$ for all $r \geq k$.

It follows from Proposition 4.1 that $Y_{r, s}(0)=D_{X_{k}}^{r, s}\left[X_{k}, J X_{k}\right](0)$ for all $r$ and $s$ such that $r+s=k-1$. Since the functions $a_{p, q}$ and $b_{p, q}$ vanish at order $k-1$ at the origin, we have for all $r$ and $s$ such that $r+s=k-1$

$$
\begin{aligned}
D_{X_{k+1}}^{r, s}\left[X_{k+1}, J X_{k+1}\right](0)= & Y_{r, s}(0)+\sum_{p+q=k-1}\left(\left(D_{X_{k}}^{r+1, s} a_{p, q}(0)-D_{X_{k}}^{r, s+1} b_{p, q}(0)\right) J Y_{p, q}(0)\right) \\
& +\sum_{p+q=k-1}\left(\left(-D_{X_{k}}^{r+1, s} b_{p, q}(0)-D_{X_{k}}^{r, s+1} a_{p, q}(0)\right) Y_{p, q}(0)\right) .
\end{aligned}
$$

Perhaps after a change of coordinates if needed, we can assume that $X_{k}=\frac{\partial}{\partial x_{1}}$ and that $J(0)$ is still the standard structure. We therefore have $J X_{k}=\sum_{k=1}^{n} J_{2 k-1,1} \frac{\partial}{\partial x_{k}}+J_{2 k} \frac{\partial}{\partial y_{k}}$ and since $J(0)$ is the standard structure, $J X_{k}(0)=\frac{\partial}{\partial y_{1}}$. Now, since $a_{p, q}$ and $b_{p, q}$ vanishes at 0 at order $k-1$, neither $X_{k}$ nor $J X_{k}$ is differentiated in $D_{X_{k}}^{r, s} a_{p, q}(0)$ and $D_{X_{k}}^{r, s} b_{p, q}(0)$ and we therefore have

$$
\begin{aligned}
& D_{X_{k+1}}^{r, s}\left[X_{k+1}, J X_{k+1}\right](0)= \\
& =Y_{r, s}(0)+\sum_{p+q=k-1}\left(\left(\frac{\partial^{k} a_{p, q}}{\partial x_{1}^{r+1} \partial y_{1}^{s}}(0)-\frac{\partial^{k} b_{p, q}}{\partial x_{1}^{r} \partial y_{1}^{s+1}}(0)\right) J Y_{p, q}(0)\right) \\
& +\sum_{p+q=k-1}\left(\left(-\frac{\partial^{k} b_{p, q}}{\partial x_{1}^{r+1} \partial y_{1}^{s}}(0)-\frac{\partial^{k} a_{p, q}}{\partial x_{1}^{r} \partial y_{1}^{s+1}}(0)\right) Y_{p, q}(0)\right) .
\end{aligned}
$$


We search for $a_{p, q}$ and $b_{p, q}$ as homogeneous polynomials in $x_{1}$ and $y_{1}$ of degree $k$ without the term $x_{1}^{k}$ such that

$$
\forall(r, s) \in \mathbb{N}, r+s=k-1,\left\{\begin{array}{l}
\frac{\partial^{k} a_{p, q}}{\partial x_{1}^{r+1} \partial y_{1}^{s}}(0)-\frac{\partial^{k} b_{p, q}}{\partial x_{1}^{r} \partial y_{1}^{s+1}}(0)=0 \\
\frac{\partial^{k} b_{r, s}}{\partial x_{1}^{r+1} \partial y_{1}^{s}}(0)+\frac{\partial^{k} a_{p, q}}{\partial x_{1}^{r} \partial y_{1}^{s+1}}(0)=0 \text { if }(r, s) \neq(p, q), \\
\frac{\partial^{k} b_{p, q}}{\partial x_{1}^{r+1} \partial y_{1}^{s}}(0)+\frac{\partial^{k} a_{p, q}}{\partial x_{1}^{r} \partial y_{1}^{s+1}}(0)=1 .
\end{array}\right.
$$

With such a choice, (6) gives $D_{X_{k+1}}^{r, s}\left[X_{k+1}, J X_{k+1}\right]=0$, for all $r$ and $s$ such that $r+s=$ $k-1$, which together with Propositions 4.1 and 4.2 proves that $X_{k+1}$ commutes at order $k+1$ at 0 . So (B) holds true.

Now, we notice that $D_{X}^{r, 0} a_{p, q}(0)=D_{X}^{r, 0} b_{p, q}(0)=0$ for all $r \in \mathbb{N}$, and all $(p, q)$. Therefore $D_{X_{k+1}}^{r, 0} X_{k+1}(0)=D_{X_{k}}^{r, 0} X_{k}(0)$ for all $r \in \mathbb{N}$, and so (国) also holds true.

We deduce from Theorem 1.4 the following generalization of Freeman's Theorem:

Theorem 4.4. If $J$ is integrable, then for all $X \in \operatorname{ker} \mathcal{L}$ and all $p$ in a neighborhood of 0 , there exists a J-holomorphic disc $\gamma$ such that $\gamma(0)=p$ and $\frac{\partial \gamma}{\partial x}(0)=X(p)$.

Proof: Let $X$ be a vector field in ker $\mathcal{L}$. We show that the Lie algebra generated by $X$ and $J X$ is included in $T^{J} M$ by proving by induction that all Lie brackets we can form with $X$ and $J X$ belong to $\operatorname{ker} \mathcal{L}$.

In the integrable case, we have the following characterization of $\operatorname{ker} \mathcal{L}$ (see [2]):

$$
\operatorname{ker} \mathcal{L}=\left\{X \in T^{J} M, \forall Y \in T^{J} M,[X, Y] \in T^{J} M\right\} .
$$

Assume that all Lie brackets of length at most $k$ belongs to ker $\mathcal{L}$. Let $X_{p}$ and $X_{q}$ be two Lie brackets of length $p$ and $q$ respectively, with $p$ and $q$ such that $p+q=k+1$. Using (7), we show that $\left[X_{p}, X_{q}\right]$ and $\left[X_{p}, J X_{q}\right]$ belong to ker $\mathcal{L}$.

Jacobi's identity gives for all $Y \in T^{J} M$ :

$$
\left[\left[X_{p}, J X_{q}\right], Y\right]=\left[X_{p},\left[J X_{q}, Y\right]\right]+\left[J X_{q},\left[Y, X_{p}\right]\right] .
$$

The vector field $J X_{q}$ belong to $\operatorname{ker} \mathcal{L}$ so (7) implies that $\left[J X_{q}, Y\right]$ belongs $T^{J} M$ and so, again (17) implies that $\left[X_{p},\left[J X_{q}, Y\right]\right]$ belongs to $T^{J} M$. Analogously, $\left[J X_{q},\left[Y, X_{p}\right]\right]$ belongs to $T^{J} M$ and so $\left[\left[X_{p}, J X_{q}\right], Y\right]$ is a element of $T^{J} M$ for all $Y$ in $T^{J} M$, which with (7) implies that $\left[X_{p}, J X_{q}\right]$ belongs to ker $\mathcal{L}$.

Analogously, $\left[X_{p}, X_{q}\right]$ belongs to ker $\mathcal{L}$. Therefore all Lie brackets of $X$ and $J X$ belong to ker $\mathcal{L}$ and so to $T^{J} M$. Applying Theorem 1.4 ends the proof of Theorem 4.4, $\square$

Another example of situation where Theorem 1.4 can be useful is the following example: 
Example 4.5. Let $\varphi: \mathbb{R}^{8} \rightarrow \mathbb{R}$ be the map defined by $\varphi\left(x_{1}, y_{1}, x_{2}, y_{2}, x_{3}, y_{3}, x_{4}, y_{4}\right)=y_{1}$ and let $M$ be the set $M=\left\{z \in \mathbb{R}^{8}, \varphi(z)=0\right\}$. We define the eight following vector fields

$$
\begin{aligned}
L_{1} & =\frac{\partial}{\partial x_{1}}, & L_{2} & =\frac{\partial}{\partial y_{1}}, \\
L_{3} & =\frac{\partial}{\partial x_{2}}-\frac{1}{2} y_{3}^{2} \frac{\partial}{\partial x_{1}}, & L_{4} & =\frac{\partial}{\partial y_{2}}+\left(-2 y_{3} x_{3}+x_{2}\right) \frac{\partial}{\partial x_{1}}, \\
L_{5} & =\frac{\partial}{\partial x_{3}}-y_{2} y_{3} \frac{\partial}{\partial x_{1}}, & L_{6} & =\frac{\partial}{\partial y_{3}}+x_{3} \frac{\partial}{\partial x_{2}}-\left(\frac{x_{3} y_{3}^{2}}{2}+x_{3} y_{2}\right) \frac{\partial}{\partial x_{1}}, \\
L_{7} & =\frac{\partial}{\partial x_{4}}+y_{4} \frac{\partial}{\partial y_{4}} & L_{8} & =\frac{\partial}{\partial y_{4}} .
\end{aligned}
$$

and the complex structure $J$ they induce by setting

$$
J L_{1}=L_{2}, \quad J L_{3}=L_{4}, \quad J L_{5}=L_{6}, \quad J L_{7}=L_{8} .
$$

This example is derived from the example of Section 2. Again $J(0)$ is the standard complex structure $J_{0}$ and the tangent space $T M$ is spanned over $\mathbb{R}$ by $L_{1}, L_{3}, L_{4}, L_{5}, L_{6}, L_{7}$ and $L_{8}$ and $T^{J} M$ is spanned over $\mathbb{C}$ by $L_{3}, L_{5}$ and $L_{7}$.

When we compute the Lie brackets of the $L_{i}$ 's in the complex tangent bundle, we get:

$$
\begin{aligned}
& {\left[L_{3}, L_{4}\right]=L_{1},} \\
& {\left[L_{3}, L_{5}\right]=0, \quad\left[L_{4}, L_{5}\right]=y_{3} \frac{\partial}{\partial x_{1}},} \\
& {\left[L_{3}, L_{6}\right]=y_{3} \frac{\partial}{\partial x_{1}}, \quad\left[L_{4}, L_{6}\right]=0, \quad\left[L_{5}, L_{6}\right]=L_{3},} \\
& {\left[L_{3}, L_{7}\right]=0, \quad\left[L_{4}, L_{7}\right]=0, \quad\left[L_{5}, L_{7}\right]=0, \quad\left[L_{6}, L_{7}\right]=0,} \\
& {\left[L_{3}, L_{8}\right]=0, \quad\left[L_{4}, L_{8}\right]=0, \quad\left[L_{5}, L_{8}\right]=0, \quad\left[L_{6}, L_{8}\right]=0, \quad\left[L_{7}, L_{8}\right]=-L_{8} .}
\end{aligned}
$$

Therefore

$$
\begin{array}{lll}
\hat{\mathcal{L}}_{\varphi}\left(L_{3}, L_{5}\right)=0, & \hat{\mathcal{L}}_{\varphi}\left(L_{3}, L_{7}\right)=0, & \hat{\mathcal{L}}_{\varphi}\left(L_{5}, L_{7}\right)=0, \\
\hat{\mathcal{L}}_{\varphi}\left(L_{3}, J L_{3}\right)=d \varphi\left(L_{2}\right)=1, & \hat{\mathcal{L}}_{\varphi}\left(L_{5}, J L_{5}\right)=d \varphi\left(L_{3}\right)=0, & \hat{\mathcal{L}}_{\varphi}\left(L_{7}, J L_{7}\right)=d \varphi\left(-L_{8}\right)=0,
\end{array}
$$

and so, $M$ is pseudoconvex and $\operatorname{ker} \mathcal{L}$ is spanned by $L_{5}$ and $L_{7}$.

As in Section 2, there is no $J$-holomorphic disc $\gamma$ included in $M$ such that $\gamma(0)=0$ and $\frac{\partial \gamma}{\partial x}(0)=\frac{\partial}{\partial x_{3}}$ but Theorem 1.4 yields the existence of a $J$-holomorphic disc $\gamma$ included in $M$, such that $\gamma(0)=0$ and $\frac{\partial \gamma}{\partial x}(0)=L_{7}(0)$.

We now prove Theorem 1.6 by applying our strategy with the following lemma:

Lemma 4.6. Let $\mathbb{L}$ be a subbundle of $T^{J} M$ such that if $X \in \mathbb{L}$ commutes at order $k$ at 0 , then for all $X_{1}, \ldots, X_{k+1} \in\{X, J X\},\left[X_{1},\left[\ldots,\left[X_{k}, X_{k+1}\right] \ldots\right]\right](0)$ belongs to $\mathbb{L}_{0}$.

Then for all $X \in \mathbb{L}$, there exists a sequence $\left(X_{k}\right)_{k}$ of vector fields in $\mathbb{L}$ such that

$(\alpha) D_{X}^{l, 0} X_{k}(0)=D_{X}^{l, 0} X(0)$ for all $k$ in $\mathbb{N}^{*}$ and $l$ in $\mathbb{N}$,

( $\beta) X_{k}$ commutes at order $k$ at 0 . 
Proof: We proceed by induction on $k$. We set $X_{1}=X$ and we assume $X_{k}$ constructed. Maybe after a change of coordinates if needed, we can assume that $X_{k}=\frac{\partial}{\partial x_{1}}$ and $J X_{k}(0)=$ $\frac{\partial}{\partial y_{1}}$. We set

$$
X_{k+1}=X_{k}+\sum_{p=1}^{\ell}\left(a_{p}+J b_{p}\right) L_{p}
$$

where $L_{1}, \ldots, L_{\ell}$ is a basis of $\mathbb{L}$ and where $a_{p}$ and $b_{p}, p=1, \ldots, \ell$, are homogeneous polynomials of degree $k$ in the variables $x_{1}$ and $y_{1}$, without the term $x_{1}^{k}$. Therefore $X_{k+1}$ belongs to $\mathbb{L}, D_{X_{k+1}}^{l, 0} X_{k+1}(0)=D_{X_{1}}^{l, 0} X_{1}(0)$ for all $l$ and $X_{k+1}$ commutes at order $k$ at 0 . Analogously to the proof of Lemma 4.3, we now choose $a_{p}$ and $b_{p}$ so that $X_{k+1}$ commutes at order $k+1$ at 0 . We compute

$$
\begin{aligned}
{\left[X_{k+1}, J X_{k+1}\right] } & =\left[X_{k}, J X_{k}\right]+\sum_{p=1}^{\ell}\left(a_{p}^{2}+b_{p}^{2}\right)\left[L_{p}, J L_{p}\right] \\
& +\sum_{p=1}^{\ell}\left(\left(X_{k}\left(a_{p}\right)-J X_{k}\left(b_{p}\right)\right) J L_{p}+\left(-X_{k}\left(b_{p}\right)-J X_{k}\left(a_{p}\right)\right) L_{p}\right) \\
& +\sum_{p=1}^{\ell}\left(a_{p}\left(\left[X_{k}, J L_{p}\right]+\left[L_{p}, J X_{k}\right]\right)-b_{p}\left(\left[X_{k}, L_{p}\right]+\left[J X_{k}, J L_{p}\right]\right)\right) \\
& +\sum_{p, q=1}^{\ell}\left(a_{q} L_{q}\left(a_{p}\right)+b_{q} J L_{q}\left(a_{p}\right)-a_{q} J L_{q}\left(b_{p}\right)+b_{q} L_{q}\left(b_{p}\right)\right) J L_{p} \\
& +\sum_{p, q=1}^{\ell}\left(-a_{q} L_{q}\left(b_{p}\right)-b_{q} J L_{q}\left(b_{p}\right)-a_{q} J L_{q}\left(a_{p}\right)+b_{q} L_{q}\left(a_{p}\right)\right) L_{p} .
\end{aligned}
$$

On the one hand, the functions $a_{p}$ and $b_{p}$ vanish at order $k-1$ at the origin. Therefore, for all $r$ and $s$ such that $r+s=k-1$

$$
\begin{aligned}
D_{X_{k+1}}^{r, s}\left[X_{k+1}, J X_{k+1}\right](0) & =D_{X_{k}}^{r, s}\left[X_{k}, J X_{k}\right](0)+\sum_{p=1}^{\ell}\left(\left(D_{X_{k}}^{r+1, s} a_{p}(0)-D_{X_{k}}^{r, s+1} b_{p}(0)\right) J L_{p}(0)\right) \\
& +\sum_{p=1}^{\ell}\left(\left(-D_{X_{k}}^{r+1, s} b_{p}(0)-D_{X_{k}}^{r, s+1} a_{p}(0)\right) L_{p}(0)\right) .
\end{aligned}
$$

On the other hand, Proposition 4.1 implies that for all $r$ and $s$ such that $r+s=k-1$

$$
D_{X_{k}}^{r, s}\left[X_{k}, J X_{k}\right](0)=[\underbrace{J X_{k},\left[\ldots,\left[J X_{k}\right.\right.}_{s}[\underbrace{X_{k}, \ldots\left[X_{k}\right.}_{r},\left[X_{k}, J X_{k}\right]] \ldots](0)
$$

and so $D_{X_{k}}^{r, s}\left[X_{k}, J X_{k}\right](0)$ belongs to $\mathbb{L}_{0}$. Hence there exists $\alpha_{1}^{r, s}, \ldots, \alpha_{\ell}^{r, s} \in \mathbb{C}$ such that

$$
D_{X_{k}}^{r, s}\left[X_{k}, J X_{k}\right](0)=\sum_{p=1}^{\ell} \alpha_{p}^{r, s} L_{p}(0)
$$


and so

$$
\begin{aligned}
D_{X_{k+1}}^{r, s}\left[X_{k+1}, J X_{k+1}\right](0) & =\sum_{p=1}^{\ell} \alpha_{p}^{r, s} L_{p}(0)+\sum_{p=1}^{\ell}\left(\left(D_{X_{k}}^{r+1, s} a_{p}(0)-D_{X_{k}}^{r, s+1} b_{p}(0)\right) J L_{p}(0)\right) \\
& +\sum_{p=1}^{\ell}\left(\left(-D_{X_{k}}^{r+1, s} b_{p}(0)-D_{X_{k}}^{r, s+1} a_{p}(0)\right) L_{p}(0)\right) .
\end{aligned}
$$

Now we choose the functions $a_{p}$ and $b_{p}$ as in Lemma 4.3 such that

$$
\forall(r, s) \in \mathbb{N}, r+s=k-1,\left\{\begin{array}{l}
\frac{\partial^{k} a_{p}}{\partial x_{1}^{r+1} \partial y_{1}^{s}}(0)-\frac{\partial^{k} b_{p}}{\partial x_{1}^{r} \partial y_{1}^{s+1}}(0)=-\operatorname{Im}\left(\alpha_{p}^{r, s}\right) \\
\frac{\partial^{k} b_{r, s}}{\partial x_{1}^{r+1} \partial y_{1}^{s}}(0)+\frac{\partial^{k} a_{p, q}}{\partial x_{1}^{r} \partial y_{1}^{s+1}}(0)=\operatorname{Re}\left(\alpha_{p}^{r, s}\right)
\end{array} .\right.
$$

After solving this system of $2 k$ equations and $2 k$ variables, we get a vector field $X_{k+1}$ such that $D_{X_{k+1}}^{r, s}\left[X_{k+1}, J X_{k+1}\right](0)=0$ for all $r$ and $s$ such that $r+s=k-1$. Since $X_{k+1}$ already commutes at order $k$, Propositions 4.1 and 4.2 then imply that in fact $X_{k+1}$ commutes at order $k+1$ at 0 .

As a corollary of Theorem 1.6, we get the following Theorem already proved by Kruzhilin and Sukhov in [5] in the smooth case:

Theorem 4.7. If $\operatorname{ker} \mathcal{L}=T^{J} M$, then for all $p \in M$ and all $v \in T^{J} M$, there exists a germ of J-holomorphic disc $\gamma$ such that $\gamma(0)=p, \frac{\partial \gamma}{\partial x}(p)=v$ and $\gamma(\mathbb{D}) \subset M$.

Proof: We prove that if $X \in T^{J} M$ commutes at order $k$ at 0 , then for all $X_{1}, \ldots, X_{k-1} \in$ $\{X, J X\},\left[X_{1},\left[\ldots,\left[X_{k-1},[X, J X]\right] \ldots\right](0)\right.$ belongs to $T_{0}^{J} M$ and we apply Theorem 1.6 . On the one hand, since $M$ is Levi flat, $[X, J X]$ belongs to $T^{J} M$ and $d \varphi(J[X, J X])$ vanishes identically.

On the other hand, since $X$ commutes at order $k$ at 0

$$
\begin{aligned}
X_{1} \cdot X_{2} \ldots X_{k-1} \cdot d \varphi(J[X, J X])(0) & =d \varphi\left(J\left(X_{1} \cdot X_{2} \ldots X_{k-1} \cdot[X, J X]\right)\right)(0) \\
& =d \varphi\left(J \left[X_{1},\left[X_{2}, \ldots\left[X_{k-1},[X, J X] \ldots\right]\right)(0)\right.\right.
\end{aligned}
$$

and so $\left[X_{1}, \ldots\left[X_{k-1},[X, J X] \ldots\right](0)\right.$ belongs to $J T_{0} M$. Considering $d \varphi([X, J X])$, one can show analogously that $\left[X_{1}, \ldots\left[X_{k-1},[X, J X] \ldots\right](0)\right.$ belongs to $T_{0} M$ and so finally to $T_{0}^{J} M$.

Let us notice that the above theorem can in fact be generalized in the following sense. If $\mathbb{L}$ is a complex bundle included in ker $\mathcal{L}$ such that

- for all $X \in \mathbb{L},[X, J X]$ belongs to $\mathbb{L}$,

- there exist $\varphi_{1}, \ldots, \varphi_{k}$ such that for all $X \in T^{J} M, X \cdot \varphi_{1}=\ldots=X \cdot \varphi_{k}=0$ if and only if $X$ belongs to $\mathbb{L}$,

then the previous proof shows that for any $X$ in $\mathbb{L}$, there exists a $J$-holomorphic disc $\gamma$ such that $\gamma(0)=p, \frac{\partial \gamma}{\partial x}(p)=v$ and $\gamma(\mathbb{D}) \subset M$. However, as we will see in the next example, Theorem 1.6 is more general. 
Example 4.8. Let $\varphi: \mathbb{R}^{8} \rightarrow \mathbb{R}$ be the map defined by $\varphi\left(x_{1}, y_{1}, x_{2}, y_{2}, x_{3}, y_{3}, x_{4}, y_{4}\right)=y_{1}$ and let $M$ be the set $M=\left\{z \in \mathbb{R}^{8}, \varphi(z)=0\right\}$. We also define the eight following vector fields

$$
\begin{aligned}
L_{1} & =\frac{\partial}{\partial x_{1}}, & L_{2} & =\frac{\partial}{\partial y_{1}}, \\
L_{3} & =\frac{\partial}{\partial x_{2}}-\frac{1}{2} y_{3}^{2} \frac{\partial}{\partial x_{1}}, & L_{4} & =\frac{\partial}{\partial y_{2}}+\left(-2 y_{3} x_{3}+x_{2}\right) \frac{\partial}{\partial x_{1}}, \\
L_{5} & =\frac{\partial}{\partial x_{3}}-y_{2} y_{3} \frac{\partial}{\partial x_{1}}, & L_{6} & =\frac{\partial}{\partial y_{3}}+x_{3} \frac{\partial}{\partial x_{2}}-\left(\frac{x_{3} y_{3}^{2}}{2}+x_{3} y_{2}\right) \frac{\partial}{\partial x_{1}}, \\
L_{7} & =\frac{\partial}{\partial x_{4}}+y_{4} \frac{\partial}{\partial y_{4}}+y_{1} y_{4} L_{5} & L_{8} & =\frac{\partial}{\partial y_{4}}+y_{1} y_{4} L_{6} .
\end{aligned}
$$

and the complex structure $J$ they induce by setting

$$
J L_{1}=L_{2}, \quad J L_{3}=L_{4}, \quad J L_{5}=L_{6}, \quad J L_{7}=L_{8} .
$$

Again this example is derived from the example of Section 2, The tangent space $T M$ is spanned over $\mathbb{R}$ by $L_{1}, L_{3}, L_{4}, L_{5}, L_{6}, L_{7}$ and $L_{8}$ and $T^{J} M$ is spanned over $\mathbb{C}$ by $L_{3}$, $L_{5}$ and $L_{7}$.

When we compute the Lie brackets of the $L_{i}$ 's in the complex tangent bundle, we get:

$$
\begin{aligned}
& {\left[L_{3}, L_{4}\right]=L_{1},} \\
& {\left[L_{3}, L_{5}\right]=0, \quad\left[L_{4}, L_{5}\right]=y_{3} \frac{\partial}{\partial x_{1}},} \\
& {\left[L_{3}, L_{6}\right]=y_{3} \frac{\partial}{\partial x_{1}}, \quad\left[L_{4}, L_{6}\right]=0, \quad\left[L_{5}, L_{6}\right]=L_{3},} \\
& {\left[L_{3}, L_{7}\right]=0, \quad\left[L_{4}, L_{7}\right]=y_{1} y_{3} y_{4} \frac{\partial}{\partial x_{1}}, \quad\left[L_{5}, L_{7}\right]=0, \quad\left[L_{6}, L_{7}\right]=y_{1} y_{4} L_{3},} \\
& {\left[L_{3}, L_{8}\right]=y_{1} y_{3} y_{4} \frac{\partial}{\partial x_{1}}, \quad\left[L_{4}, L_{8}\right]=0, \quad\left[L_{5}, L_{8}\right]=y_{1} y_{4} L_{3}, \quad\left[L_{6}, L_{8}\right]=0,}
\end{aligned}
$$

and

$$
\left[L_{7}, L_{8}\right]=-y_{1} L_{5}+y_{1}^{2} y_{4}^{2} L_{3}+2 y_{1} y_{4} L_{6}-L_{8} .
$$

Therefore

$$
\begin{aligned}
\hat{\mathcal{L}}_{\varphi}\left(L_{3}, L_{5}\right) & =0 \\
\hat{\mathcal{L}}_{\varphi}\left(L_{3}, L_{7}\right) & =0 \\
\hat{\mathcal{L}}_{\varphi}\left(L_{5}, L_{7}\right) & =0 \\
\hat{\mathcal{L}}_{\varphi}\left(L_{3}, J L_{3}\right) & =d \varphi\left(L_{2}\right)=1 \\
\hat{\mathcal{L}}_{\varphi}\left(L_{5}, J L_{5}\right) & =d \varphi\left(L_{3}\right)=0 \\
\hat{\mathcal{L}}_{\varphi}\left(L_{7}, J L_{7}\right) & =d \varphi\left(y_{1}^{2} y_{4}^{2} L_{4}-y_{1} y_{4} L_{5}-y_{1} L_{6}-L_{8}\right)=0
\end{aligned}
$$


and so, $M$ is pseudoconvex and $\operatorname{ker} \mathcal{L}$ is spanned by $L_{5}$ and $L_{7}$. As in Section 2 , there is no $J$-holomorphic disc $\gamma$ included in $M$ such that $\gamma(0)=0$ and $\frac{\partial \gamma}{\partial x}(0)=\frac{\partial}{\partial x_{3}}$. However, Theorem 1.6 can be applied to $L_{7}$ with $\mathbb{L}$ the subbundle generated by $L_{7}$. In order to do this, it suffices to check that for all $X_{1}, \ldots, X_{k} \in\left\{L_{7}, L_{8}\right\}$, the Lie bracket $\left[X_{1},\left[\ldots,\left[X_{k},\left[L_{7}, L_{8}\right] \ldots\right](0)\right.\right.$ is a linear combination of $L_{7}$ and $L_{8}$. By induction on $k$, we get

$$
\left[X_{1},\left[\ldots,\left[X_{k},\left[L_{7}, L_{8}\right] \ldots\right]=y_{1} L+\alpha L_{8}\right.\right.
$$

where $L$ is a vector field and $\alpha$ belongs to $\{-1,0,1\}$, both depending on the sequence $X_{1}, \ldots, X_{k}$. Therefore $\left[X_{1},\left[\ldots,\left[X_{k},\left[L_{7}, L_{8}\right] \ldots\right](0)\right.\right.$ belongs to the subbundle generated by $L_{7}$ at 0 and Theorem [1.6 yields the existence of a $J$-holomorphic disc $\gamma$ included in $M$, such that $\gamma(0)=0$ and $\frac{\partial \gamma}{\partial x}(0)=L_{7}(0)$.

\section{REFERENCES}

[1] J.-F. Barraud, E. Mazzilli: Regular type of real hyper-surface in (almost) complex manifolds, Math. Z. 248, 757-772 (2004)

[2] M. Freeman: Local Complex foliation of Real Submanifolds, Math. Ann. 209, 1-30 (1974).

[3] S. Ivashkovich, J.-P. Rosay: Schwarz-type lemmas for solutions of $\bar{\partial}$-inequalities and complete hyperbolicity of almost complex manifolds, Ann. Inst. Fourier (Grenoble) 54 (2004), no. 7, 2387-2435 (2005).

[4] S. Ivashkovich, A. Sukhov: Schwarz reflection principle, boundary regularity and compactness for $J$-complex curves, Ann. Inst. Fourier 60, no. 4, 1489-1513 (2010).

[5] N. Kruzhilin, A. Sukhov: Pseudoholomorphic discs attached to CR-submanifolds of almost complex spaces, Bull. Sci. Math. 129, no. 5, 398-414 (2005).

[6] L. H. Loomis: An introduction to abstract harmonic analysis, D. Van Nostrand Company, Inc., Toronto-New York-London, (1953).

[7] J.-C. Sikorav: Some properties of holomorphic cueves in almost complex manifolds, Prog. Math. 117, 165-189, Birkhauser, Basel (1994).

Laboratoire Paul Painlevé U.M.R. CNRS 8524, U.F.R. De Mathématiques, cité scientifique, Université Lille 1, F59 655 Villeneuve d’Ascq Cedex, France.

E-mail address: william.alexandre@math.univ-lille1.fr

Laboratoire Paul Painlevé U.M.R. CNRS 8524, U.F.R. De Mathématiques, Cité scientifique, Université Lille 1, F59 655 Villeneuve D'AscQ Cedex, France.

E-mail address: emmanuel.mazzilli@math.univ-lille1.fr 